\title{
On increasing the noise immunity of micro acoustic-mechanical gyroscope
}

\author{
I. P. Miroshnichenko ${ }^{1, *}$, I. A. Parinov ${ }^{2}$, and V. P. Sizov ${ }^{1}$ \\ ${ }^{1}$ Don State Technical University, Rostov-on-Don, Russia \\ ${ }^{2}$ Southern Federal University, Rostov-on-Don, Russia
}

\begin{abstract}
The paper proposes and describes a new modified micro acoustic-mechanical gyroscope, which, while maintaining the positive qualities of known analogues, differs in comparison with them its enhanced functionality by converting the angular speeds of rotation of the supporting base into electrical signals simultaneously with respect to two axes of rotation and increasing the level of the useful signal compared to with noise level. The proposed technical solution is protected by a patent of the Russian Federation for invention. The proposed gyroscope can be used in navigation systems, orientation and control of various moving objects in aviation technology, auto transport, robotics, etc.
\end{abstract}

\section{Introduction}

Currently, in the navigation systems, orientation and control of various moving objects in aeronautical engineering, automobile transport, robotics and other technical objects, micro acoustic-mechanical gyroscopes are successfully used, which allows reducing mass and geometrical characteristics, increasing reliability, increasing operating life, reducing production cost of the pointed objects [1-5].

Micromechanical gyroscopes are known [6-9], a significant shortcoming of which, hindering their practical application, is the limitation of their functional capabilities, due to these devices provide registration of the angular velocity of rotation of the supporting base only with respect to one direction of its rotation. At the same time, to register angular velocities with respect to two directions of rotation of the supporting base, it is necessary to additionally install a second similar gyroscope. It leads to a complication of the construction and increase the device cost. Significant shortcoming of the gyroscopes [6-9] is also the low level of the useful signal, compared with the level of noise, which leads to the need to use recording equipment with high sensitivity, which also increases the cost of the device.

A new micro acoustic-mechanical gyroscope was proposed in $[10,11]$, a theoretical calculation of which was presented in [12].

This gyroscope contains a supporting base, a regular structure of inertial masses (RSIMs) placed in a chess order, active piezoelectric transducers (APTs) and measuring interdigital transducers (MIDTs) of the total field of a surface acoustic wave (SAW) from the RSIMs, consisting of diffraction and SAW signal fields from Coriolis forces. The base is made of isotropic material, on its outer surface there is a thin film of a piezoelectric material with RSIMs and MIDTs installed on it for each direction of rotation, with the MIDTs placed symmetrically relative to the position of the RSIMs and perpendicular to the axes of rotation of the base.

A trapezoidal ledge is made on the inner surface of the supporting base, the larger base of the ledge is faced towards the outer surface of the carrying base.

APTs are installed symmetrically to each other on the side surfaces of the trapezoidal ledge and provide the excitation of longitudinal acoustic waves in the material of the supporting base in the directions determined by the angle $\mathrm{Q}$, defined as.

$$
\sin Q=v_{l} / v_{R}
$$

where $v_{l}$ is the velocity of longitudinal waves in the material of the supporting base $1, v_{R}$ is the velocity of the SAWs, and the angle $Q$ is defined by the position of the side surfaces of the trapezoidal ledge relative to the outer surface of the base.

The technical solution $[10,11]$ allows for the conversion of angular velocities of rotation of the supporting base into electrical signals simultaneously with respect to two axes of its rotation, however, significant drawback of this solution remains the low level of the useful signal as compared to the level of noise hindrances.

\section{Formulation of the problem}

The objectives of the proposed technical solution were to expand the functionality by converting the angular velocities of rotation of the supporting base into electric signals simultaneously with respect to the two axes of its rotation and increasing the level of the useful signal compared to the level of noise hindrances.

\footnotetext{
* Corresponding author: ipmir2011@yandex.ru
} 


\section{Description of technical solution}

To achieve these goals, a new modified, noise-free micro acoustic-mechanical gyroscope has been developed, the essence of which is illustrated by graphic materials shown in Figures 1-5, where Figure 1 shows schematically the proposed gyroscope (top view), Figure 2 demonstrates section A-A, Figure 3 presents the proposed gyroscope (bottom view), in Figs. 4 and 5 there are schematics of the electrical connections of the MIDTs and APTs, respectively

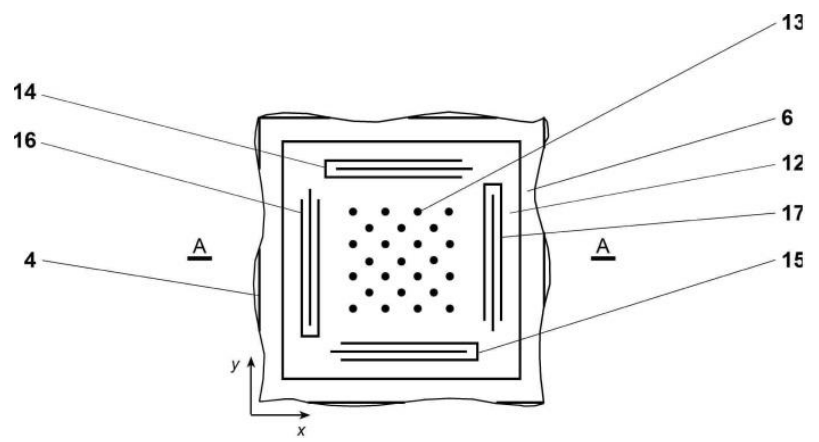

Fig. 1. Scheme of the proposed gyroscope (top view).

The proposed gyroscope (Figure 2) contains a supporting base 1 made of an isotropic material, on the outer 2 and inner 3 surfaces of which trapezoidal ledges 4 and 5 are made, respectively, coinciding in shapes and sizes and placed mirror relative to each other.

The ledge 4 has a small base 6 , a larger base 7 and side surfaces 8 , while the larger base 7 faces the inner surface 3 of the base 1 . The ledge 5 has a small base 9 , a larger base 10 and side surfaces 11 , while the larger base 10 is faced towards the outer surface 2 of the base 1 .

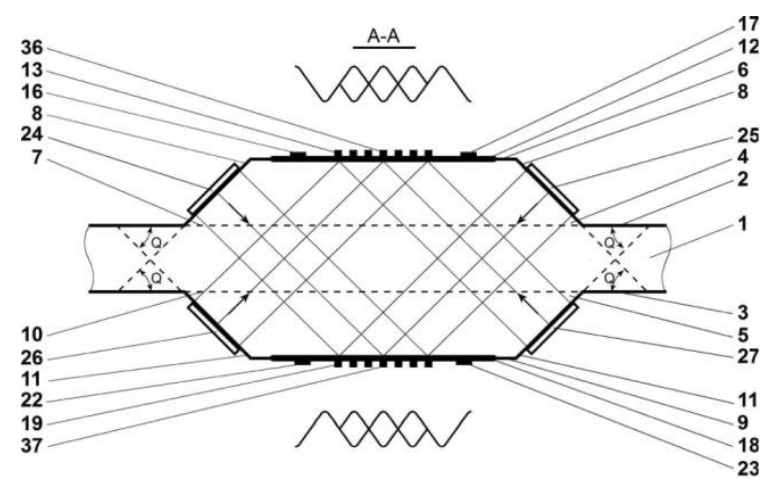

Fig. 2. Scheme of the proposed gyroscope (section A-A).

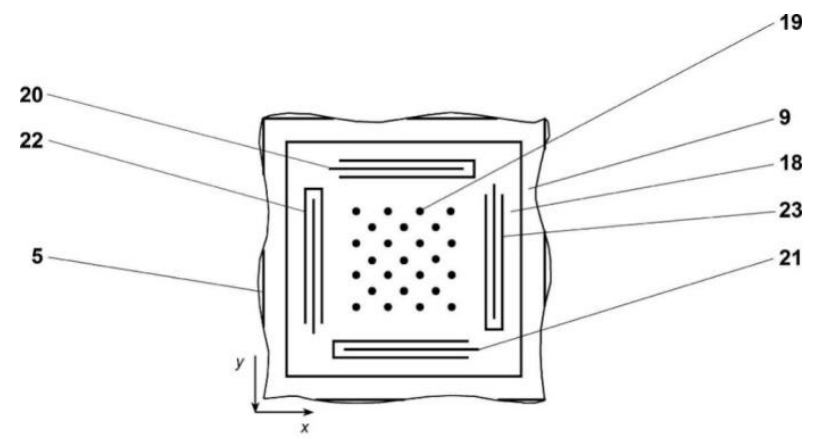

Fig. 3. Scheme of the proposed gyroscope (bottom view).
On the surface of the small base 6 of the ledge 4, a thin film 12 of a piezoelectric material is installed with RSIM 13 and MIDTs 14, 15 installed (along $x$-axis) and 16, 17 (along $y$-axis) (Figure 1) of the summary SAWs field from RSIM 13, consisting of diffraction and signal fields of SAWs from Coriolis forces. On the surface of the small base 9 of the ledge 5 (Figure 2), a thin film 18 of a piezoelectric material is installed with RSIM 19 and MIDTs 20, 21 installed (along the $x$-axis) and 22, 23 (along the $y$-axis) (Figure 3 ) of the summary SAWs field from RSIM 19, consisting of diffraction and signal fields of SAWs from Coriolis forces. The side surfaces 8 of the ledge 4 and the side surfaces 11 of the ledge 5 (Figure 2) form, in accordance with the inner surface 3 and the outer surface 2 of the base 1 , angle $Q$, which is chosen from the condition of optimal excitation of Rayleigh waves (1) on them. The angle $Q$ is defined by the position of the side surfaces 8 and 11, respectively, of the ledges 4 and 5 relative to the surfaces 3 and 2 of the base 1 . APTs 24 and 25 are installed symmetrically on the side surfaces 8 of the ledge 4, and APPs 26 and 27 are installed symmetrically on the side surfaces 11 of the ledge 5 , which excite the longitudinal acoustic waves in the base material in the directions determined by the angle Q.

APTs 26 and 27 (Figure 5) are electrically coupled to generator G28, and APTs 24 and 25 are electrically joined to generator G28 and inverter I29, while APTs 24 and 25 provide excitation in antiphase with respect to APTs 26 and 27 .

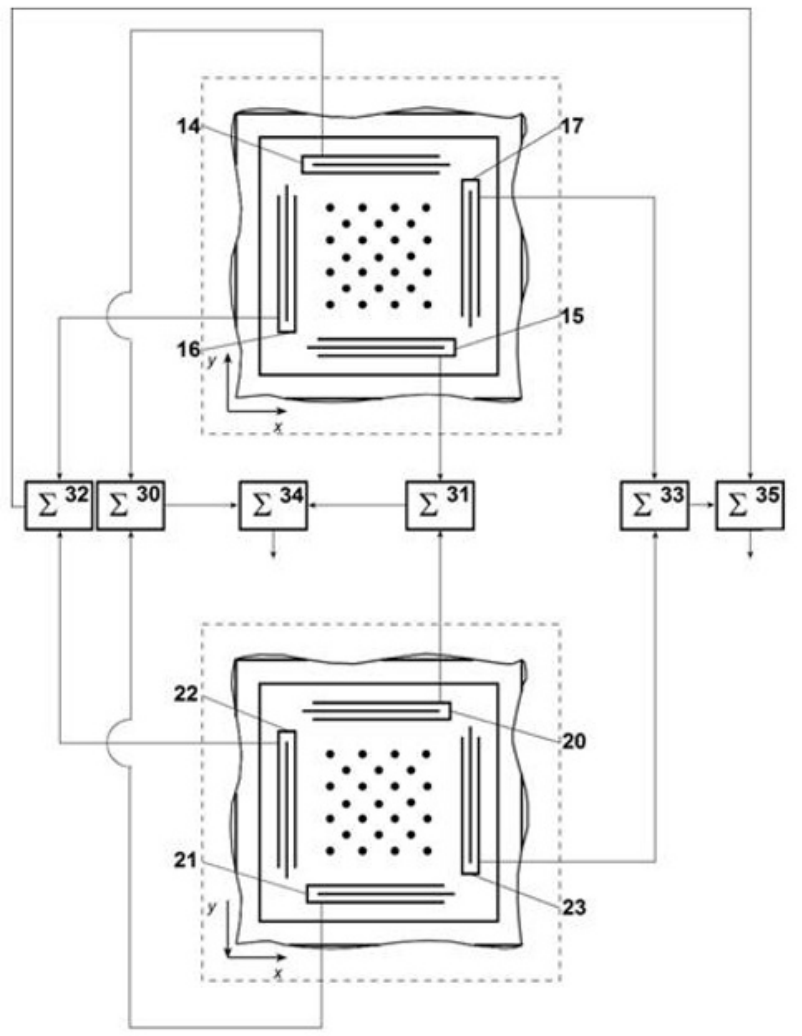

Fig. 4. Scheme of electrical connections of MIDTs.

MIDTs 14, 15, placed on the surface of the small base 6 of the ledge 4 (along the $x$-axis), and MIDTs 16, 17 (along 
the $y$-axis) are mounted on a thin film 12 symmetrically relative to the position of RSIM 13 and perpendicular to the axes of rotation of the base 1 . MIDTs 20, 21, placed on the surface of the small base 9 of the ledge 5 (Figure 3) (along the $x$-axis), and MIDTs 22, 23 (along the $y$-axis) are mounted on a thin film 18 symmetrically relative to the position of RSIM 19 and perpendicular to the axes of rotation of the base 1 (Figure 2). The outputs of the MIDTs 14 and 20,15 and 21, 16 and 22, 17 and 23 are electrically coupled in pairs with the inputs of the adders $30,31,32$ and 33 , respectively (Figure 4). The outputs of the adders 30 and 31, 32 and 33 are joined, respectively, to the inputs of the adders 34 and 35. RSIMs 13 and 19 are located in chess order with distances between them, providing preferential radiation in the directions to the MIDTs. The outputs of the adders 34 and 35 are joined to the registration system (not shown in the figures).

The proposed gyroscope operates as follows.

With the help of generator G28 and inverter I29, APTs 26, 27 and 24,25 excite in the supporting base 1 longitudinal waves, which, by interacting with the side surface 8 of the small base 6 of the ledge 4 and the side surface 11 of the small base 9 of the ledge 5, excite Rayleigh waves running into different sides along the $x$ axis, while APTs 24 and 25 provide excitation in antiphase with respect to APTs 26 and 27.

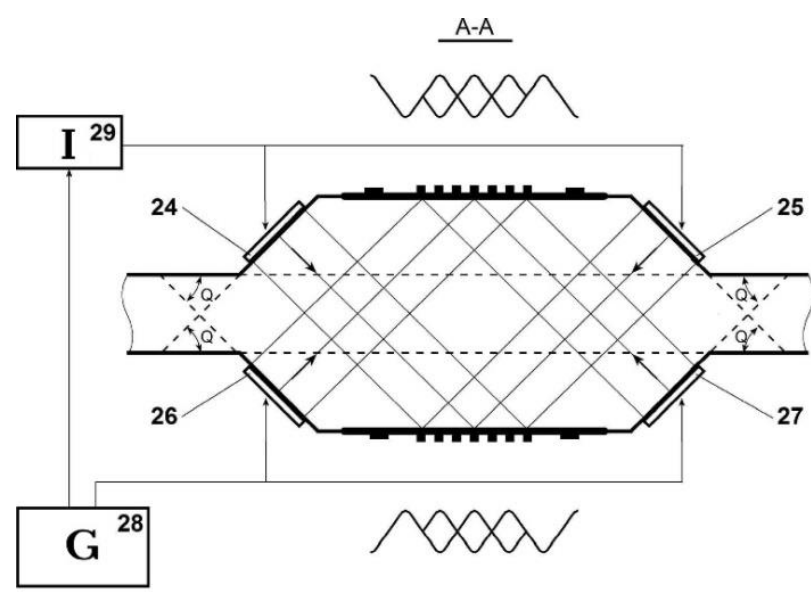

Fig. 5. Scheme of electrical connections of APTs.

In the areas 36 and 37 of the interference of the beams of longitudinal waves, respectively, on the surface 8 of the small base 6 of the ledge 4 and the surface 11 of the small base 9 of the ledge 5 , standing waves are formed with distances between antinodes equal to: $\lambda_{R} / 2$, where $\quad \lambda_{R}=v_{R} / f ; f$ is the excitation frequency.

In these areas are placed RSIMs 13 and 19, which allow us to increase the Coriolis force arising during the rotation of the gyroscope.

Under the influence of standing waves, the RSIMs 13 and 19 make vertical (along $z$-axis) oscillations. The oscillating masses, in turn, are the sources of SAWs that propagate along the $x$ - and $y$-axes. Thus, from areas 36 and 37 of the interference of longitudinal wave beams, where RSIMs 13 and 19 are located, in the direction of the MIDTs 14, 15, 16, 17, 20, 21, 22, 23 traveling waves are propagated, which these MIDTs detect. As a result, the corresponding signals appear at the outputs of the MIDTs.

When the gyroscope rotates around the $x$-axis, the Coriolis force, directed along the $y$-axis, acts on the inertial mass structures 13 and 19 moving along the $z$ axis:

$$
F=2 m[\Omega V],
$$

where $m$ is the mass of the oscillating structure; $\Omega$ is the angular velocity of rotation of the gyroscope; $\mathrm{V}$ is the oscillatory mass velocity.

Under the influence of this force, an additional SAW is generated, which changes the electrical signal at the outputs of the MIDTs 16, 17 and 22, 23. This change is proportional to the angular velocity $\Omega$ directed along the $x$ axis. At the outputs of the MIDTs 14, 15 and 20, 21, the signal remains practically unchanged.

When the gyroscope rotates around the $y$-axis, similar phenomena occur, and a useful signal arises at the outputs of the MIDTs 14, 15 and 20, 21.

With simultaneous rotation of the gyroscope around the $x$ - and $y$-axes, a useful signal appears on all the MIDTs, and the level of the signals at the outputs of the MIDTs 16, 17 and 22, 23 corresponds to the speed of rotation around the $x$-axis, and the level of the signals at the outputs MIDTs 14, 15 and 20, 21 corresponds to the speed of rotation around the $y$-axis. Thus, signals arise that allow one to judge on the rotation of the base 1 with respect to two axes. The signals from the outputs of the MIDTs 14 and 21 arrive to the inputs of the adder 30, from the outputs of the MIDTs 15 and 20 arrive to the inputs of the adder 31, from the outputs of the MIDTs 16 and 22 arrive to the inputs of the adder 32, and from the outputs of the MIDTs 17 and 23 arrive to the inputs of the adder 33. Here the summation is performed from the outputs of the corresponding MIDTs. The signals arising under the action of SAWs (excited by the corresponding waves, which are emitted by the APTs 24,25 and 26, 27 at the output of the each of adders 30, 31 and 32,33) decrease. It occurs because the transducers 24,25 , and 26, 27 , respectively, excite waves in antiphase, but signals, arising under the action of additional SAWs at the rotation of the base 1 , are in phase (since the Coriolis acceleration of all masses has the same direction) and increase at the outputs of these adders.

The signals from the outputs of the adders 30 and 31 arrive to the inputs of the adder 34 , but the signals from the outputs of the adders 32 and 33 arrive to the inputs of the adder 35 . Here it is also produced the summation of signals from all the MIDTs, corresponding to the rotation axes of the base 1 . Then the signals from the outputs of the adders 34 and 35 arrive to the registration system. In this case, a significant increase in the level of the useful signal is achieved compared to the level of noise hindrances.

\section{Conclusions}

A new modified, noise-free micro acoustic-mechanical gyroscope, which preserves the positive qualities of the known analogues, is developed. In comparison with 
them, it expands the functionality by converting the angular speeds of rotation of the supporting base into electrical signals simultaneously with respect to two axes of its rotation and increasing the level of the useful signal compared to the level of noise hindrances.

The proposed gyroscope is protected by a patent of the Russian Federation for an invention [13] and can be applied in navigation systems, orientation and control of various mobile objects in aviation technology, auto transport, robotics, etc.

The work was supported by the grant of Russian Foundation for Basic Research, No. 19-08-00365, Ministry of Education and Science of the Russian Federation: projects Nos. 3.5378.2017/VU (organization of the implementation of researches), 9.9770.2017/BCh (the basic part of the state task).

\section{References}

[1] V.A. Matveev, Gyroscope is Eeasy. Moscow: Bauman MSTU Press.- 191 (2012)

[2] D.P. Lukyanov, Yu.V. Filatov, S.Yu. Shevchenko, The current state and prospects for the development of solid-state microgyroscopes on the base of surface acoustic waves Gyroscopy and navigation 3(74), 75-87 (2011)

[3] M.I. Evstifeev, Main stages of the development of domestic micromechanical gyroscopes News of universities. Instrument making 6, 75-80 (2011)

[4] V.A. Kalinin, Y.V. Lavrov, V.A. Melnikov, V.A. Shubarev, Mathematical modeling of gyroscope on SAWS Electronics: Science, technology, business. Special issue 47-51 (2008)

[5] V.K. Varadan, V.V. Varadan, Microsensors, microelectromechanical systems (MEMS), and electronics for smart structures and systems Smart Mater. Struct. 9, 953-972 (2009)

[6] V.A. Kalinin, V.D. Lukyanov, V.A. Shubarev, V.A. Melnikov, Piezoelectric gyroscope Patent RU 2387951, 27.04.2010.

[7] V.A. Kalinin, V.D. Lukyanov, V.A. Shubarev, V.A. Melnikov, Gyroscope on the base of surface acoustic waves Patent RU No. 2390727, 27.05.2010

[8] R. Aigner, Apparatus and method for detecting a rotation Patent US No.7895892, 01.03.2011.

[9] V.K. Varadan, P.B. Xavier, W.D. Suh, J.A. Kollakompil, V.V. Varadan, Micro-Electromechanical Gyroscope Patent US 6984332, 10.01.2006.

[10] Y.V. Vahtin, I.P. Miroshnichenko, V.P. Sizov, V.A. Pogorelov, Micro acoustic-mechanical gyroscope Patent RU 2543706, 10.03.2015.

[11] I.P. Miroshnichenko, V.A. Pogorelov, V.P. Sizov, Modified micromechanical Gyroscope Bulletin of the Don State Technical University 2, 73-77 (2015)

[12] I.P. Miroshnichenko, V.A. Pogorelov, V.P. Sizov, The method of scalarization in problems of the propagation of surface elastic waves in a rotating transversely isotropic half-space Bulletin of the Don State Technical University 3, 7-18 (2015)
[13] I.P. Miroshnichenko, A.C. Mitkin, V.P. Sizov, V.A. Pogorelov, Modified micro acousticmechanical gyroscope Patent RU 2582483, 27.04.2016. 